\title{
A Computational Study of the Formation and Dimerization of
}

\author{
Benzothiet-2-one \\ Dhandapani V. Sadasivam and David M. Birney* \\ Department of Chemistry and Biochemistry \\ Texas Tech University, Lubbock, TX 79409-1061 \\ david.birney@ttu.edu
}

\section{Supporting Information}

\section{Contents}

Absolute energies from B3LYP calculations, for structures

related to the formation and reaction of 4 , Table S1

S2

Energetics of C1-S bond stretching of 4, Figure S1

S3

Selected B3LYP/6-31G(d,p) molecular orbitals of TS8a, Figure S2

S4

Additional computational details

S4

Complete Gaussian 03 Reference

S5

Cartesian coordinates for all stationary points, Table S2

S6 
Table S1. Absolute energies (Hartree), zero point vibrational energies (ZPVE, $\mathrm{kcal} / \mathrm{mol}$ ), low or imaginary frequencies $\left(\mathrm{cm}^{-1}\right)$ and dipole moments (Debye) for structures related to the formation and reactions of 4 as calculated at the B3LYP/6-31G(d,p) level of theory.

\begin{tabular}{ccccc} 
Structure & $\begin{array}{c}\text { Absolute energy } \\
\text { (Hartree) }\end{array}$ & $\begin{array}{c}\text { ZPVE } \\
\mathrm{kcal} / \mathrm{mol}\end{array}$ & $\begin{array}{c}\text { freq } \\
\left(\mathrm{cm}^{-1}\right)\end{array}$ & $\begin{array}{c}\text { dipole } \\
\text { (Debye) }\end{array}$ \\
\hline $\mathbf{2}$ & -855.89680 & 62.6 & 80.9 & 5.31 \\
CO & -113.30495 & 3.2 & 2208.6 & 0.06 \\
$\mathbf{3}$-DMSO & -742.54147 & 55.0 & 92.6 & 8.61 \\
$\mathbf{4}$ & -742.54530 & 56.1 & 129.3 & 3.77 \\
$\mathbf{5 a}$ & -1485.11410 & 113.5 & 40.0 & 0.00 \\
$\mathbf{5 b}$ & -1485.13750 & 114.2 & 43.0 & 6.71 \\
$\mathbf{5 c}$ & -1485.11990 & 113.9 & 40.6 & 1.41 \\
TS6 & -855.82701 & 59.8 & $253.4 \mathrm{i}$ & 4.71 \\
$\mathbf{7 a}$ & -1485.05693 & 110.9 & 11.3 & 0.01 \\
$\mathbf{7 c}$ & -1485.05680 & 111.1 & 16.2 & 4.62 \\
TS8a & -1485.06138 & 111.8 & $309.5 \mathrm{i}$ & 0.00 \\
TS8b & -1485.05880 & 111.6 & $315.2 \mathrm{i}$ & 6.86 \\
TS8c & -1485.05075 & 111.2 & $128.4 \mathrm{i}$ & 0.31
\end{tabular}

3-DMSO was calculated using a dielectric continuum model with the dielectric corresponding to DMSO. 


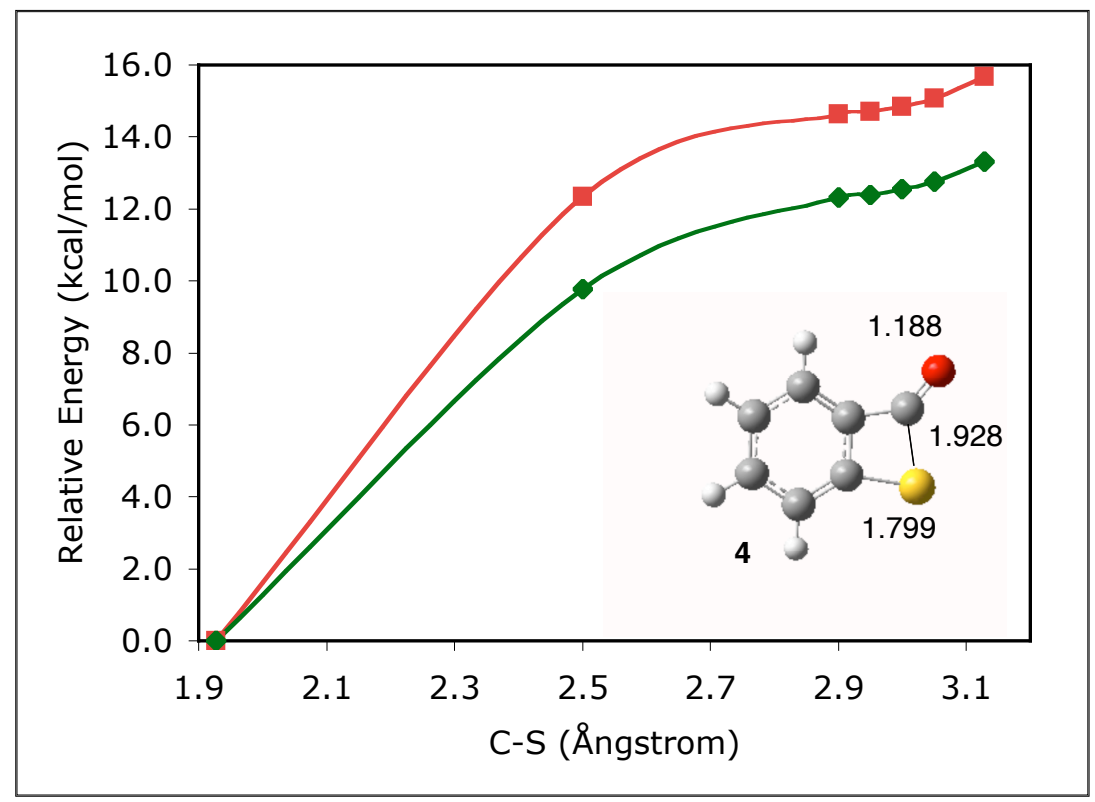

Figure S1. Relative energies from constrained optimizations of 4, stretching the C1-S bond from $1.928 \AA$ towards hypothetical 3. Green data are at the B3LYP/6-31G $(\mathrm{d}, \mathrm{p})+$ ZPVE level of theory and red are at the G3MP2B $3^{\text {S1 }}$ level. The line is a 4th order polynomial fit. The structure shown is the B3LYP/6-31G(d,p) optimized geometry of 4 . 

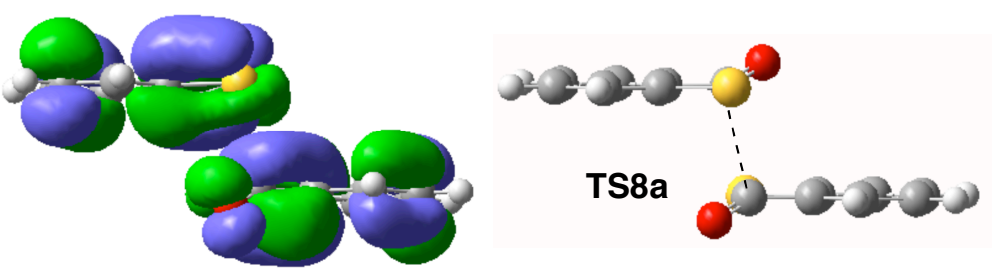

LUMO
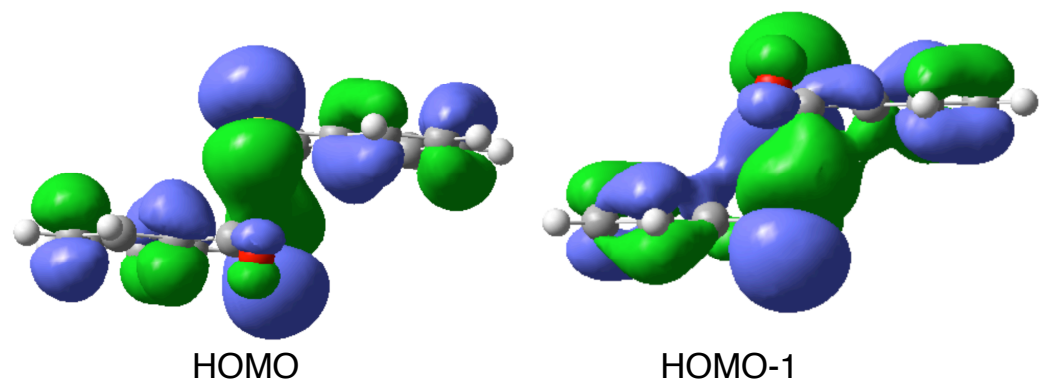

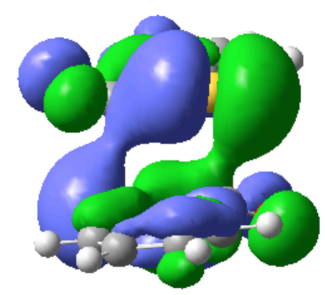

HOMO-2

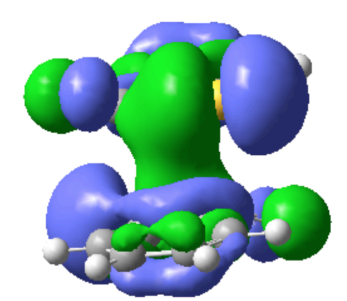

HOMO-3

Figure S2. Surface of the selected frontier molecular orbitals of TS8a from the B3LYP/6-31G(d,p) wavefunction. These are viewed from different perspectives to highlight the significant features of the MO's.

\section{Additional computational details:}

All structures were fully optimized using Gaussian03 ${ }^{\mathrm{S} 2}$ at the B3LYP/6-31G(d,p) level of theory, unless otherwise noted. Frequency calculations verified the identity of each stationary point as a minimum or a transition state with zero or one imaginary frequency 
respectively. Zero-point vibrational energy corrections have been applied to the B3LYP energies, but have not been scaled. Intrinsic reaction coordinate (IRC) ${ }^{\mathrm{S} 3}$ calculations were performed to connect the transition state TS8c to the complex 7c and to the product $\mathbf{5 c}$. The energy scans in Figure S1 were performed by constraining the C1-S distance and optimizing the rest of the structure. The calculation of 3 -DMSO was carried out using the Polarizable Continuum model using the integral equation formalism mode (IEF-PCM) in which the cavity is created via a series of overlapping spheres. The dielectric constant of DMSO was used $(\mathrm{e}=46.7)$.

\section{References:}

S1. Baboul, A. G.; Curtiss, L. A.; Redfern, P. C.; Raghavachari, K., J. Chem. Phys. 1999, $110,(16), 7650-7657$.

S2. Complete Gaussian 03 Reference

Gaussian 03, Revision C.02, Frisch, M. J.; Trucks, G. W.; Schlegel, H. B.; Scuseria, G. E.; Robb, M. A.; Cheeseman, J. R.; Montgomery, Jr., J. A.; Vreven, T.; Kudin, K. N.; Burant, J. C.; Millam, J. M.; Iyengar, S. S.; Tomasi, J.; Barone, V.; Mennucci, B.; Cossi, M.; Scalmani, G.; Rega, N.; Petersson, G. A.; Nakatsuji, H.; Hada, M.; Ehara, M.; Toyota, K.; Fukuda, R.; Hasegawa, J.; Ishida, M.; Nakajima, T.; Honda, Y.; Kitao, O.; Nakai, H.; Klene, M.; Li, X.; Knox, J. E.; Hratchian, H. P.; Cross, J. B.; Bakken, V.; Adamo, C.; Jaramillo, J.; Gomperts, R.; Stratmann, R. E.; Yazyev, O.; Austin, A. J.; Cammi, R.; Pomelli, C.; Ochterski, J. W.; Ayala, P. Y.; Morokuma, K.; Voth, G. A.; Salvador, P.; Dannenberg, J. J.; Zakrzewski, V. G.; Dapprich, S.; Daniels, A. D.; Strain, M. C.; Farkas, O.; Malick, D. K.; Rabuck, A. D.; Raghavachari, K.; Foresman, J. B.; Ortiz, J. V.; Cui, Q.; Baboul, A. G.; Clifford, S.; Cioslowski, J.; Stefanov, B. B.; Liu, G.; Liashenko, A.; Piskorz, P.; Komaromi, I.; Martin, R. L.; Fox, D. J.; Keith, T.; Al-Laham, M. A.; Peng, C. Y.; Nanayakkara, A.; Challacombe, M.; Gill, P. M. W.; Johnson, B.; 
Chen, W.; Wong, M. W.; Gonzalez, C.; and Pople, J. A.; Gaussian, Inc., Wallingford CT, 2004.

S3. Fukui, K. Acc. Chem. Res. 1981, 14, 363-368. 
Table S2. Cartesian coordinates of all stationary structures at the B3LYP/6-

$31 \mathrm{G}(\mathrm{d}, \mathrm{p})$ level of theory.

Carbon monoxide

$\begin{array}{lrrr}\mathrm{C} & .000000 & .000000 & -.650254 \\ \mathrm{O} & .000000 & .000000 & .487691 \\ & & & \\ \mathbf{2} & & & \\ \mathrm{C} & .404711 & .636790 & .000047 \\ \mathrm{C} & .521244 & -.763125 & .000086 \\ \mathrm{C} & 1.775844 & -1.364592 & .000276 \\ \mathrm{C} & 2.909808 & -.545246 & .000426 \\ \mathrm{C} & 2.802029 & .850928 & .000388 \\ \mathrm{C} & 1.543652 & 1.446801 & .000197 \\ \mathrm{C} & -.978669 & 1.121641 & -.000158 \\ \mathrm{C} & -1.973393 & -.088880 & -.000281 \\ \mathrm{H} & 1.876219 & -2.445133 & .000308 \\ \mathrm{H} & 3.892936 & -1.006831 & .000576 \\ \mathrm{H} & 3.697489 & 1.463688 & .000507 \\ \mathrm{H} & 1.420838 & 2.525642 & .000163 \\ \mathrm{O} & -1.381815 & 2.263849 & -.000232 \\ \mathrm{O} & -3.167429 & .001219 & -.000453 \\ \mathrm{~S} & -1.032805 & -1.651488 & -.000123\end{array}$

\section{3-DMSO}

C -0.060442

C -0.071020

C 1.116862

C 2.327616

C 2.374953

C 1.234626

H 1.033219

H 3.250214

H 3.347675

H 1.306997

C -1.299034

S -1.529395

O $\quad-2.276145$

$\begin{array}{lll}-0.748585 & 0.000069\end{array}$

$0.695352-0.000226$

$1.502909-0.000651$

$0.864919-0.000343$

$\begin{array}{ll}-0.557785 & 0.000520\end{array}$

$\begin{array}{ll}-1.334639 & 0.000756\end{array}$

$2.588117-0.001178$

$1.440058-0.000681$

$\begin{array}{ll}-1.048600 & 0.000939\end{array}$

$\begin{array}{ll}-2.420092 & 0.001303\end{array}$

$\begin{array}{ll}1.300826 & 0.000118\end{array}$

$-1.633727-0.000587$

$1.905270 \quad 0.000943$

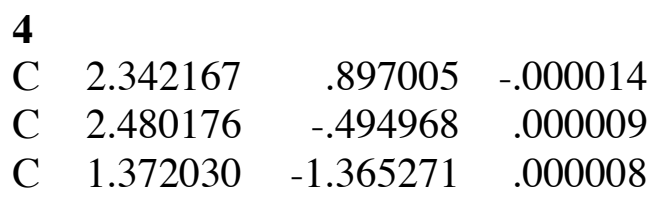

$\begin{array}{lrrr}\mathrm{C} & .127854 & -.765574 & .000000 \\ \mathrm{C} & -.012627 & .630751 & .000014 \\ \mathrm{C} & 1.071412 & 1.494237 & -.000016 \\ \mathrm{~S} & -1.611452 & -1.225532 & -.000020 \\ \mathrm{C} & -1.495700 & .698808 & .000003 \\ \mathrm{O} & -2.336485 & 1.538703 & .000024 \\ \mathrm{H} & 3.229953 & 1.520872 & -.000133 \\ \mathrm{H} & .948082 & 2.572188 & .000039 \\ \mathrm{H} & 3.478764 & -.922886 & .000083 \\ \mathrm{H} & 1.506443 & -2.441216 & .000112\end{array}$

\section{$5 \mathbf{a}$}

$\begin{array}{llll}\text { C } & -1.924098 & .661493 & -.384743\end{array}$

$\begin{array}{llll}\text { C } & -1.957613 & -.737350 & -.232791\end{array}$

S $\quad-.436580 \quad 1.406259-1.063594$

C $\quad-.690378 \quad-1.521487 \quad-.443016$

$\begin{array}{llll}\text { O } & -.424767 & -2.189906 & -1.400139\end{array}$

$\begin{array}{llll}\text { C } & 1.957657 & .737347 & .232838\end{array}$

$\begin{array}{llll}\text { C } & 1.924073 & -.661496 & .384740\end{array}$

$\begin{array}{llll}\text { S } & .436508 & -1.406155 & 1.063615\end{array}$

$\begin{array}{llll}\text { C } & .690474 & 1.521530 & .443164\end{array}$

$\begin{array}{llll}\text { O } & .424931 & 2.189990 & 1.400268\end{array}$

$\begin{array}{llll}\text { C } & -3.077262 & 1.410895 & -.151108\end{array}$

$\begin{array}{llll}\text { C } & -3.142346 & -1.375185 & .136696\end{array}$

$\begin{array}{llll}\text { C } & -4.290593 & -.616263 & .377218\end{array}$

$\begin{array}{llll}\mathrm{H} & -5.211076 & -1.112757 & .668135\end{array}$

$\begin{array}{llll}\text { C } & -4.256660 & .770572 & .239401\end{array}$

$\begin{array}{llll}\mathrm{H} & -5.149635 & 1.358526 & .427656\end{array}$

$\begin{array}{llll}\text { C } & 3.142404 & 1.375135 & -.136687\end{array}$

$\begin{array}{llll}\text { C } & 3.077169 & -1.410963 & .151001\end{array}$

$\begin{array}{llll}\text { C } & 4.256580 & -.770691 & -.239555\end{array}$

H $\quad 5.149508 \quad-1.358688 \quad-.427894$

$\begin{array}{llll}\text { C } & 4.290589 & .616150 & -.377310\end{array}$

$\begin{array}{llll}\text { H } & 5.211085 & 1.112599 & -.668261\end{array}$

$\begin{array}{llll}\mathrm{H} & -3.048318 & 2.487094 & -.282632\end{array}$

$\begin{array}{llll}\mathrm{H} & -3.164919 & -2.455723 & .240024\end{array}$

H $3.048169 \quad-2.487165 \quad .282479$

H $3.165034 \quad 2.455675 \quad-.239972$

$5 \mathbf{b}$

$\begin{array}{lllll}\text { O } & -2.164334 & 1.943890 & -1.772534\end{array}$

$\begin{array}{llll}\text { C } & 1.305407 & .976863 & -.027152\end{array}$

$\begin{array}{llll}\text { C } & 1.724100 & -.366396 & -.040372\end{array}$

$\begin{array}{llll}\text { O } & 2.164553 & -1.942733 & -1.773580\end{array}$

C $\quad 1.348857-1.343590-1.113503$

C $-1.348688 \quad 1.344211-1.112876$ 


$\begin{array}{lrrr}\mathrm{S} & .407608 & 1.728571 & -1.392841 \\ \mathrm{~S} & -.407396 & -1.728023 & -1.393591 \\ \mathrm{C} & -1.724053 & .366403 & -.040347 \\ \mathrm{C} & -1.305333 & -.976860 & -.027690 \\ \mathrm{C} & 1.735039 & 1.816619 & 1.007769 \\ \mathrm{C} & 2.572150 & -.838844 & .968676 \\ \mathrm{C} & 2.556067 & 1.329843 & 2.024750 \\ \mathrm{H} & 2.872977 & 1.992781 & 2.823947 \\ \mathrm{C} & 2.978159 & .000745 & 2.003814 \\ \mathrm{H} & 3.629714 & -.379909 & 2.784169 \\ \mathrm{C} & -2.572324 & .838332 & .968763 \\ \mathrm{C} & -1.735148 & -1.817110 & 1.006758 \\ \mathrm{C} & -2.556375 & -1.330842 & 2.023821 \\ \mathrm{H} & -2.873409 & -1.994169 & 2.822646 \\ \mathrm{C} & -2.978509 & -.001751 & 2.003429 \\ \mathrm{H} & -3.630228 & .378518 & 2.783836 \\ \mathrm{H} & -2.908742 & 1.869347 & .930429 \\ \mathrm{H} & -1.429252 & -2.857950 & 1.000185 \\ \mathrm{H} & 1.429155 & 2.857465 & 1.001640 \\ \mathrm{H} & 2.908545 & -1.869852 & .929927\end{array}$

$5 \mathbf{c}$

$\begin{array}{lrrr}\mathrm{C} & -1.973142 & -.621041 & -.425904 \\ \mathrm{C} & -1.883673 & .632472 & .218358 \\ \mathrm{C} & -.603773 & 1.352439 & .468942 \\ \mathrm{~S} & -.548011 & -1.523655 & -1.006435 \\ \mathrm{O} & -.334575 & 1.897912 & 1.507502 \\ \mathrm{O} & .333903 & -1.897560 & 1.507614 \\ \mathrm{C} & .603615 & -1.352249 & .469116 \\ \mathrm{C} & 1.883583 & -.632450 & .218509 \\ \mathrm{C} & 1.973278 & .621064 & -.425757 \\ \mathrm{~S} & .548334 & 1.523930 & -1.006336 \\ \mathrm{C} & -3.235445 & -1.192261 & -.630496 \\ \mathrm{C} & -3.051492 & 1.250528 & .696593 \\ \mathrm{C} & -4.300157 & .685831 & .464915 \\ \mathrm{H} & -5.196827 & 1.185769 & .816635 \\ \mathrm{C} & -4.389119 & -.533314 & -.211901 \\ \mathrm{H} & -5.358679 & -.988757 & -.389839 \\ \mathrm{C} & 3.051330 & -1.250733 & .696656 \\ \mathrm{C} & 3.235679 & 1.192076 & -.630319 \\ \mathrm{C} & 4.389247 & .532931 & -.211754 \\ \mathrm{H} & 5.358880 & .988221 & -.389683 \\ \mathrm{C} & 4.300082 & -.686237 & .464991 \\ \mathrm{H} & 5.196672 & -1.186362 & .816647 \\ \mathrm{H} & 2.956403 & -2.190411 & 1.230788 \\ \mathrm{H} & 3.303659 & 2.166986 & -1.101720\end{array}$

$\begin{array}{rrrr}\mathrm{H} & -3.303270 & -2.167171 & -1.101918 \\ \mathrm{H} & -2.956709 & 2.190178 & 1.230802\end{array}$

\section{TS6}

$\begin{array}{lrrr}\mathrm{C} & .447183 & .674533 & -.189958 \\ \mathrm{C} & .434187 & -.767477 & -.224539 \\ \mathrm{C} & 1.669388 & -1.388690 & .122650 \\ \mathrm{C} & 2.800230 & -.658876 & .410884 \\ \mathrm{C} & 2.792261 & .760810 & .403096 \\ \mathrm{C} & 1.631113 & 1.427013 & .115034 \\ \mathrm{C} & -.693544 & 1.438426 & -.374851 \\ \mathrm{C} & -2.234160 & .080537 & .714151 \\ \mathrm{H} & 1.694239 & -2.472842 & .130577 \\ \mathrm{H} & 3.722935 & -1.180524 & .648563 \\ \mathrm{H} & 3.699085 & 1.314834 & .619873 \\ \mathrm{H} & 1.590200 & 2.511456 & .106003 \\ \mathrm{O} & -1.326827 & 2.343506 & -.730233 \\ \mathrm{O} & -3.263713 & -.186581 & 1.140248 \\ \mathrm{~S} & -.941380 & -1.676624 & -.665246\end{array}$

7a

$\begin{array}{llll}\text { C } & 2.220017 & 0.955351 & 0.134941\end{array}$

$\begin{array}{llll}C & 1.677132 & -0.097050 & 0.962991\end{array}$

$\begin{array}{llll}\text { S } & 1.474717 & 2.480589 & 0.120954\end{array}$

$\begin{array}{llll}\text { C } & 0.602170 & 0.260103 & 1.734417\end{array}$

$\begin{array}{llll}\text { O } & -0.281560 & 0.403837 & 2.460624\end{array}$

C $-1.676384 \quad 0.096236-0.963251$

$\begin{array}{llll}\text { C } & -2.220778 & -0.954488 & -0.134132\end{array}$

S $-1.475510 \quad-2.479738-0.116144$

C $-0.600071 \quad-0.262641-1.732028$

$\begin{array}{llll}\text { O } & 0.284949 & -0.407630 & -2.456430\end{array}$

$\begin{array}{llll}\text { C } & 3.368110 & 0.585400 & -0.629767\end{array}$

$\begin{array}{llll}\text { C } & 2.202058 & -1.434660 & 1.001013\end{array}$

$\begin{array}{llll}\text { C } & 3.295277 & -1.714092 & 0.231173\end{array}$

$\begin{array}{llll}\mathrm{H} & 3.720466 & -2.711387 & 0.227883\end{array}$

$\begin{array}{llll}\text { C } & 3.876121 & -0.690534 & -0.578400\end{array}$

$\begin{array}{llll}\mathrm{H} & 4.747279 & -0.936718 & -1.179413\end{array}$

$\begin{array}{llll}\text { C } & -2.201184 & 1.433766 & -1.004895\end{array}$

$\begin{array}{llll}\text { C } & -3.370183 & -0.583052 & 0.627839\end{array}$

$\begin{array}{llll}\text { C } & -3.878087 & 0.692793 & 0.572996\end{array}$

$\begin{array}{llll}\mathrm{H} & -4.750317 & 0.940189 & 1.171955\end{array}$

$\begin{array}{llll}\text { C } & -3.295801 & 1.714724 & -0.237584\end{array}$

$\begin{array}{llll}\mathrm{H} & -3.721000 & 2.712020 & -0.237045\end{array}$

H $3.821337 \quad 1.343082-1.259941$

H $1.724131 \quad-2.186531 \quad 1.618429$

$\begin{array}{llll}\mathrm{H} & -3.824555 & -1.339465 & 1.258711\end{array}$ 


\begin{tabular}{|c|c|c|c|c|c|c|c|}
\hline \multirow[t]{2}{*}{$\mathrm{H}$} & \multirow[t]{2}{*}{-1.722159} & \multirow{2}{*}{\multicolumn{2}{|c|}{$2.184404-1.622964$}} & $\mathrm{H}$ & -5.120154 & 1.471536 & 499317 \\
\hline & & & & $\mathrm{C}$ & 3.243855 & 1.365596 & -.303718 \\
\hline \multicolumn{4}{|c|}{ 7c } & $\mathrm{C}$ & 3.139366 & -1.416432 & .331955 \\
\hline $\mathrm{C}$ & -2.011577 & .194708 & -.814110 & $\mathrm{C}$ & 4.265533 & -.842122 & -.268922 \\
\hline $\mathrm{C}$ & -1.953447 & -.295246 & .544364 & $\mathrm{H}$ & 5.119362 & -1.472325 & -.500488 \\
\hline $\mathrm{C}$ & -1.670983 & -1.628539 & .683854 & $\mathrm{C}$ & 4.324913 & .524373 & -.578957 \\
\hline $\mathrm{S}$ & -1.660300 & -.842830 & -2.110571 & $\mathrm{H}$ & 5.219785 & .932334 & -1.037481 \\
\hline $\mathrm{O}$ & -1.489713 & -2.734787 & .948069 & $\mathrm{H}$ & -3.112807 & 2.472905 & -.574529 \\
\hline $\mathrm{O}$ & 1.489096 & -2.735697 & -.946672 & $\mathrm{H}$ & -3.269962 & -2.424458 & .538513 \\
\hline $\mathrm{C}$ & 1.670632 & -1.629384 & -.682948 & $\mathrm{H}$ & 3.112207 & -2.473184 & .574170 \\
\hline $\mathrm{C}$ & 1.953484 & -.296097 & -.544089 & $\mathrm{H}$ & 3.270167 & 2.424141 & -.538924 \\
\hline $\mathrm{C}$ & 2.011472 & .194885 & .814051 & & & & \\
\hline $\mathrm{S}$ & 1.659600 & -.841457 & 2.111278 & \multicolumn{4}{|c|}{ TS8b } \\
\hline $\mathrm{C}$ & -2.369890 & 1.572603 & -.936857 & $\mathrm{O}$ & -2.189565 & -2.392280 & -1.082919 \\
\hline $\mathrm{C}$ & -2.178740 & .528279 & 1.699677 & $\mathrm{C}$ & 1.315612 & .116426 & -1.045594 \\
\hline $\mathrm{C}$ & -2.500114 & 1.840922 & 1.500189 & $\mathrm{C}$ & 1.854701 & -.065821 & .234942 \\
\hline $\mathrm{H}$ & -2.677879 & 2.495179 & 2.346322 & $\mathrm{O}$ & 2.192607 & -2.390503 & 1.081435 \\
\hline $\mathrm{C}$ & -2.600645 & 2.352513 & .170530 & $\mathrm{C}$ & 1.657589 & -1.474171 & .585200 \\
\hline $\mathrm{H}$ & -2.863496 & 3.398217 & .035196 & $\mathrm{C}$ & -1.655402 & -1.475354 & -.586905 \\
\hline $\mathrm{C}$ & 2.179324 & .526540 & -1.699948 & $\mathrm{~S}$ & .674673 & -1.425033 & -1.599342 \\
\hline $\mathrm{C}$ & 2.370164 & 1.572771 & .935836 & $\mathrm{~S}$ & -.673487 & -1.425681 & 1.600570 \\
\hline $\mathrm{C}$ & 2.601415 & 2.351784 & -.172069 & $\mathrm{C}$ & -1.854307 & -.067710 & -.235182 \\
\hline $\mathrm{H}$ & 2.864547 & 3.397512 & -.037454 & $\mathrm{C}$ & -1.316357 & .114491 & 1.045870 \\
\hline $\mathrm{C}$ & 2.501051 & 1.839229 & -1.501364 & $\mathrm{C}$ & 1.378965 & 1.379407 & -1.637214 \\
\hline $\mathrm{H}$ & 2.679223 & 2.492795 & -2.347944 & $\mathrm{C}$ & 2.461558 & .954394 & .966396 \\
\hline $\mathrm{H}$ & 2.084868 & .101627 & -2.692916 & $\mathrm{C}$ & 1.985783 & 2.414379 & -.919589 \\
\hline $\mathrm{H}$ & 2.443575 & 1.987078 & 1.935693 & $\mathrm{H}$ & 2.045994 & 3.402868 & -1.365865 \\
\hline $\mathrm{H}$ & -2.443419 & 1.986183 & -1.937007 & $\mathrm{C}$ & 2.521519 & 2.211271 & .361282 \\
\hline \multirow[t]{2}{*}{$\mathrm{H}$} & -2.084160 & .104068 & 2.692933 & $\mathrm{H}$ & 2.989956 & 3.038136 & .885096 \\
\hline & & & & $\mathrm{C}$ & -2.461482 & .952223 & -.966842 \\
\hline \multicolumn{2}{|c|}{ TS8a } & & & $\mathrm{C}$ & -1.381617 & 1.377384 & 1.637687 \\
\hline $\mathrm{C}$ & -2.057333 & .584352 & -.612111 & $\mathrm{C}$ & -1.988889 & 2.411983 & 919993 \\
\hline $\mathrm{C}$ & -2.127810 & -.775302 & -.284677 & $\mathrm{H}$ & -2.050482 & 3.400240 & 1.366603 \\
\hline $\mathrm{S}$ & -.488652 & .864610 & -1.366823 & $\mathrm{C}$ & -2.523339 & 2.208828 & -.361424 \\
\hline $\mathrm{C}$ & -.848360 & -1.361721 & -.708368 & $\mathrm{H}$ & -2.992228 & 3.035363 & -.885351 \\
\hline $\mathrm{O}$ & -.409003 & -2.322770 & -1.219956 & $\mathrm{H}$ & -2.866571 & .775629 & -1.957442 \\
\hline $\mathrm{C}$ & 2.127923 & .775306 & .284784 & $\mathrm{H}$ & -.980095 & 1.547580 & 2.630849 \\
\hline $\mathrm{C}$ & 2.057274 & -.584333 & .612317 & $\mathrm{H}$ & .976501 & 1.549539 & -2.630002 \\
\hline $\mathrm{S}$ & .488758 & -.864212 & 1.367523 & $\mathrm{H}$ & 2.867603 & .777947 & 1.956624 \\
\hline $\mathrm{C}$ & .848642 & 1.361999 & .708696 & & & & \\
\hline $\mathrm{O}$ & .409623 & 2.323301 & 1.220131 & \multicolumn{2}{|c|}{ TS8c } & & \\
\hline $\mathrm{C}$ & -3.139794 & 1.416154 & -.332293 & $\mathrm{C}$ & -1.988602 & -.413007 & .859765 \\
\hline $\mathrm{C}$ & -3.243816 & -1.365894 & .303375 & $\mathrm{C}$ & -2.375432 & .415008 & -.246919 \\
\hline $\mathrm{C}$ & -4.325191 & -.524943 & .578196 & $\mathrm{C}$ & -1.536829 & 1.441864 & -.654817 \\
\hline $\mathrm{H}$ & -5.220151 & -.933135 & 1.036339 & $\mathrm{~S}$ & -.529118 & -.182855 & 1.717126 \\
\hline $\mathrm{C}$ & -4.266052 & .841560 & .268140 & $\mathrm{O}$ & -1.200944 & 2.503148 & -.966867 \\
\hline
\end{tabular}




$\begin{array}{lrrr}\mathrm{O} & 1.201195 & 2.503933 & .964850 \\ \mathrm{C} & 1.537063 & 1.442431 & .653561 \\ \mathrm{C} & 2.375527 & .415154 & .246506 \\ \mathrm{C} & 1.988479 & -.413894 & -.859328 \\ \mathrm{~S} & .528823 & -.184616 & -1.716613 \\ \mathrm{C} & -2.924365 & -1.429650 & 1.207393 \\ \mathrm{C} & -3.601087 & .240156 & -.964679 \\ \mathrm{C} & -4.455068 & -.759939 & -.578208 \\ \mathrm{H} & -5.392682 & -.910348 & -1.101837 \\ \mathrm{C} & -4.103492 & -1.595146 & .515294 \\ \mathrm{H} & -4.784288 & -2.388284 & .811610 \\ \mathrm{C} & 3.601343 & .241012 & .964175 \\ \mathrm{C} & 2.924178 & -1.430866 & -1.206179 \\ \mathrm{C} & 4.103446 & -1.595691 & -.514163 \\ \mathrm{H} & 4.784187 & -2.389107 & -.809859 \\ \mathrm{C} & 4.455245 & -.759442 & .578476 \\ \mathrm{H} & 5.392971 & -.909345 & 1.102049 \\ \mathrm{H} & 3.835947 & .898670 & 1.794661 \\ \mathrm{H} & 2.673127 & -2.082834 & -2.035771 \\ \mathrm{H} & -2.673474 & -2.080828 & 2.037655 \\ \mathrm{H} & -3.835520 & .897014 & -1.795847\end{array}$

